\title{
Molecular estimation of alteration in intestinal microbial composition in Hashimoto's thyroiditis patients
}

Hafiz Muhammad Ishaq, Imran Shair Mohammad, Hui Guo, Muhammad Shahzad, Yin Jian Hou, Chaofeng Ma, Zahid Naseem, Xiaokang Wu, Peijie Shi and Jiru Xu

This is the accepted manuscript $\odot$ 2017, Elsevier Licensed under the Creative Commons AttributionNonCommercial-NoDerivatives 4.0 International: http://creativecommons.org/licenses/by-nc-nd/4.0/

\section{(cc) BY-NC-ND}

The published article is available from doi: https://doi.org/10.1016/j.biopha.2017.08.101 
3 Hafiz Muhammad Ishaq ${ }^{\mathrm{a}}$, Imran Shair Mohammad ${ }^{\mathrm{b}}, \mathrm{Hui}_{\mathrm{Guo}}^{\mathrm{c}}$, Muhammad Shahzad ,

4 Yin Jian $\mathrm{Hou}^{\mathrm{e}}$, Chaofeng $\mathrm{Ma}^{\mathrm{f}}$, Zahid Naseem ${ }^{\mathrm{g}}$, Xiaokang $\mathrm{Wu}^{\mathrm{h}}$, Peijie Shi ${ }^{\mathrm{c}}$, Jiru $\mathrm{Xu}^{\mathrm{a} *}$

5 a Department of Pathogenic Microbiology and Immunology, School of Basic Medical

6 Sciences, Xi'an Jiaotong University, Xi'an, China.

7 bepartment of Pharmaceutics, School of Pharmacy, China Pharmaceutical University,

8 Nanjing, China.

$9 \quad{ }^{\mathrm{c}}$ Department of Endocrinology and metabolic diseases, 1st affiliated Hospital Xi'an Jiotong

10 University, China

$11{ }^{\mathrm{d}}$ Department of Pharmacology, University of Health Sciences, Punjab, Lahore, Pakistan.

$12{ }^{\mathrm{e} C e l l u l a r}$ and Molecular Biology Center, China Pharmaceutical University, Nanjing, China

$13{ }^{\mathrm{f}} \mathrm{Xi}$ 'an center for disease control and prevention, China.

$14{ }^{\mathrm{g}}$ School of Science, Engineering, and Technology Abertay University Bell street, Dundee, 15 UK.

$16{ }^{\mathrm{h}}$ Thesecond Affiliated Hospital of Xi'an Jiaotong University, 157 Xiwu Street, Xi'an

17 China.

18 For correspondence: Prof. Dr. Jiru Xu

19 Department of Pathogenic Microbiology and Immunology, School of Basic Medical 20 Sciences, Xi'an Jiaotong University, Xi'an, China.

21 Email:xujiru@mail.xjtu.edu.cn

22

23

24 Abstract 
25 The gut microbiotahasa crucial effect on human health and physiology. Hypothyroid 26 Hashimoto's thyroiditis(HT) is an autoimmune disorder manifested with environmental

27 and genetic factors. However, it is hypothesized that intestinal microbes might play avital

28 role in the pathogenesis of HT. The aim of current study was to investigate and characterize

29 the gut microbial composition of HT patients both quantitatively and qualitatively. The

30 fecal samples from 29 HT patients and 12 healthy individuals were collected. The PCR-

31 DGGE targeted V3 siteof 16S rRNA gene and real time PCR for Bifidobacterium

32 Lactobacillus, Bacteroides vulgatus and Clostridium leptumwereperformed.

33 Pyrosequencing of 16S rRNA gene with V4 location was performedon 20 randomly

34 selected samples. The comparative analysis of diversity and richness indices revealed

35 diversification of gut microbiota in HT as compared to control. The statistical data

36 elucidate the alterations in phyla of HT patients which was also affirmed at the family level.

37 We observed the declined abundance ofPrevotella_9 and Dialister, while elevated genera

38 of the diseased group included Escherichia-Shigella and Parasutterella. The alteration in

39 gut microbial configuration was also monitored at the species level,which showed an

40 increased abundance of E.coliin HT. Therefore, the current study is in agreement with the

41 hypothesis that HT patients have intestinal microbial dysbiosis. The taxa statistics at

42 species-level along with each gut microbial community were modified in HT. Thus, the

43 current study may offer the new insights into the treatment of HT patients, disease pathway, 44 and mechanism.

45 Keywords: Hashimoto's thyroiditis, Gut microbiota, Hyporthyroidism, DGGE, 46 Pyrosequencing

47 At a glance 


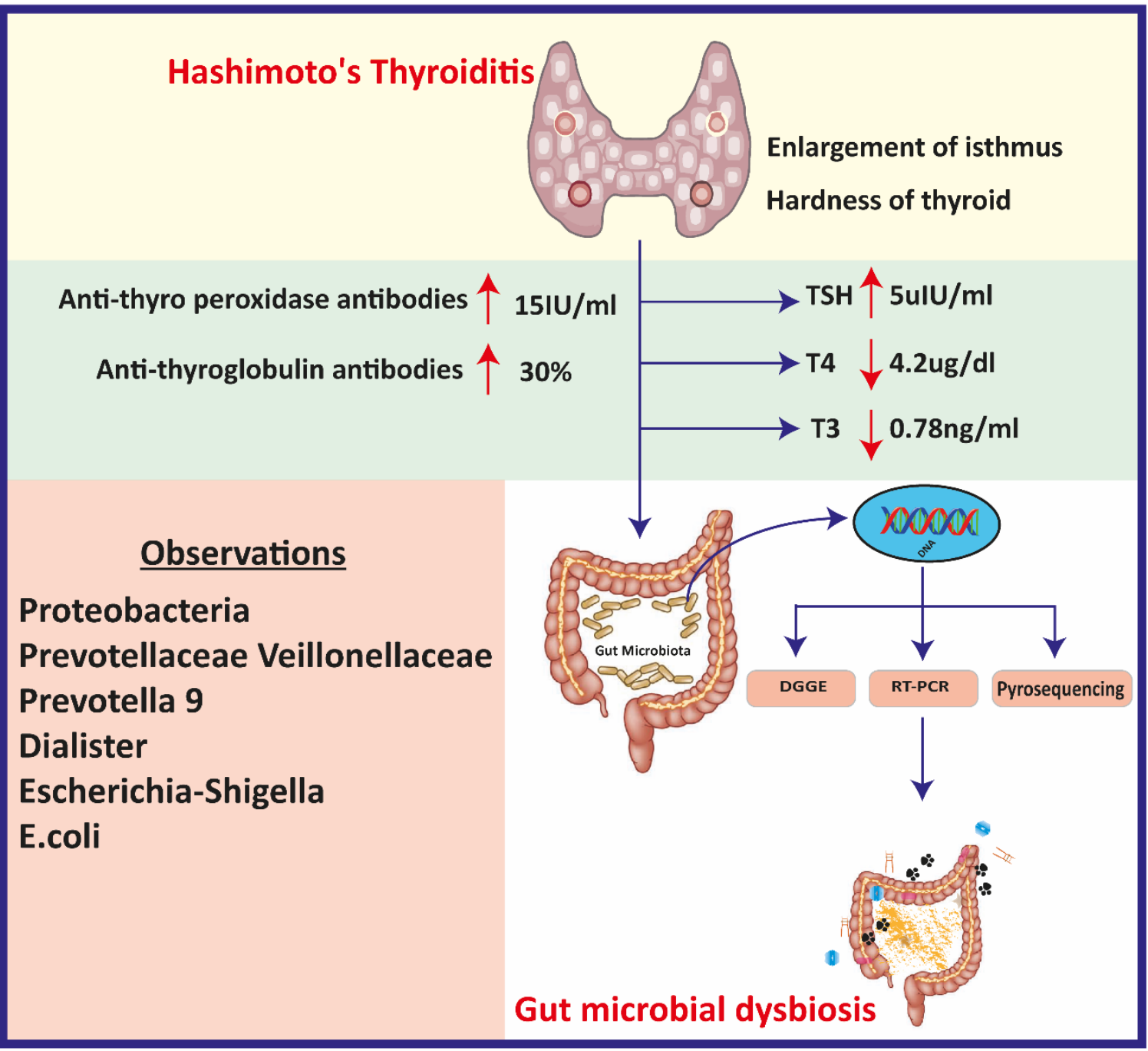

1. Introduction

50 The human gut microbiotais a major factor for host health status, and its contribution is

51 crucial for normal body mechanism, thus considered as vital aspect for influencing the

52 health grading of an individual [1]. The complexion of the human gut microbiota is quite

53 diverse with approximately 100 trillion microbes in the body serve as a metabolic,

54 nutrition, absorption and immune function against pathogens [2]. The abnormality in the

55 body homeostasis can, in turn, affect the composition pattern of gut microbiota,therefore

56 resulting in diseases implications[3]. 
$57 \quad$ HT is specifically organ linked autoimmune disease characterized by thyroid gland chronic

58 inflammation. The disease was first reported in 1912 by Hakaru Hashimoto and was

59 referred as autoimmune thyroid deficient disease (AITD).The exact pathogenicity of the

60 disease still needs to be unraveled under the intense phase of most probable

61 mechanisms[4]. The disease is now believed to be the autoimmune [5] endocrine disorder

62 [6] considered as the contributing factor of hypothyroidism [7].

63 This autoimmune disorder manifested with no unusual clinical symptoms but with the

64 gradual deterioration of thyroid gland, characterized by goiter, hypothyroidism, weight

65 gain, constipation, and depression [8].

66 The epidemiological data depicted the prevalence of disease frequency eight times higher

67 in females as compared to males [9]. The most common cause of hypothyroidism is iodine

68 deficiency[10-12].The body's innate immune mechanism permits the binding of specific

69 receptors thus identifying the molecules related to gut bacteria. The specificity of bound

70 receptors activate the immune response of the host and release the defensive cytokines,

71 white blood cells and peptides [13].

72 The recent molecular studies performed on 250 Chinese HT patients identified that single

73 nucleotide polymorphism (SNP) in STAT3 gene has an association with HT [14]. The

74 bacteriocins production by intestinal bacteria competing for nutrients and clinging of gut

75 lining thus averting any colonization by pathogens [15].

76 The modulation in gut floral configuration has been linkedto numerous disease disorders,

77 including colitis, Crohn's disease, viral diarrhea, metabolic diseases like obesity, and

78 diabetes type II [16]. Mori $\mathrm{K}$ et al. described the possible relationship between thyroid

79 autoimmunity and gut with weak evidence having very few studies consolidating such link. 
80 Furthermore, the review emphasizes to validate the hypothesis of gut microbial dysbiosis

81 in HT with further research[17].

82 The current study aim was to estimate the alteration, similarity, and diversity of gut

83 microbiota quantitatively and qualitatively in HT patients in comparison to healthy

84 controls. By using PCR-DGGE and sensitive metagenomic pyrosequencing, we have

85 monitored the gut microbial similarity and diversity in patients suffering from HT disease.

86 The investigations demonstrated the variation in bacterial taxa richness in contrast to

87 controls, with some distinct gut microbes depicting significantly higher or lower abundance

88 against the control. The significance of these alterations in the gut microbiota of HT was

89 notably high-pitched as never reported before regarding gut microbial characterization in

90 HT. Current findings thus help to illustrate the overall composition of gut microbiota in

91 HT patients.

92 2. Material and methods

$93 \quad$ 2.1. Ethics statement

94 The informed written consent was obtained from all the participants of the study including 95 diseased patients as well as healthy volunteers. Moreover, the study was approved by an 96 institutional ethical review committee of Xián Jiaotong University and performed under 97 the guidelines of the World Medical Association and Declaration of Helsinki.

\section{$98 \quad$ 2.2.Sample collection}

99 Fecal samples from 29 HT patients (20 females and 9 males, aged between 40 to 60 years) 100 and 12 healthy volunteers ( 8 females 4 males, aged between 40 to 60 years) were collected 101 in a sterile cup. The patients with HT were diagnosed according to the standard protocol of 102 department of endocrinology and metabolic diseases, $1^{\text {st }}$ affiliated hospital, school of 
103 medicine Xi' an Jiaotong University. It includes goiter, especially the enlargement of the

104 isthmus, medium hardness of the enlarged thyroid. The Thyroid stimulating

105 hormone(TSH) was higher than $5 \mu \mathrm{IU} / \mathrm{ml}$, T4 was lower than $4.2 \mu \mathrm{g} / \mathrm{dl}$, T3 was lower than

$1060.78 \mathrm{ng} / \mathrm{ml}$, anti-thyroid peroxidase antibodies level was higher than $15 \mathrm{IU} / \mathrm{ml}$, and anti-

107 thyroglobulin antibodies was more than 30\%[18]. Normal range of serum thyroid

108 hormones and antibodies are TSH $(0.25-5 \mu \mathrm{IU} / \mathrm{ml}), \mathrm{T} 4(4.2-13.5 \mu \mathrm{g} / \mathrm{dl}), \mathrm{T} 3(0.78-2.20$

$109 \mathrm{ng} / \mathrm{ml})$, Anti-TPOAb $(<15 \mathrm{IU} / \mathrm{ml})$ and Anti-TGAb $(<30 \%)$.A questionnaire for each

110 participant was prepared based on the information about gender, age, body weight, health

111 and dietary habits. All the samples were delivered on ice, usually within 4 hours of

112 defecation. Upon arrival in the laboratory, the fecal samples were stored at $-80^{\circ} \mathrm{C}$ until

113 DNA extraction. Neither of the patients and healthy individuals had any history of 114 gastrointestinal diseases nor taken antibiotics, probiotics, and prebiotics, 60 days before 115 sampling.

\subsection{DNA extraction from fecal sample}

117 QIAGEN (Hilden, Germany) Stool kit was used for DNA extraction after thawing the fecal

118 samples, with the first step of bead-beating at $5000 \mathrm{rpm}$ for $30 \mathrm{~s}$. Nano Photometer

119 (IMPLEN, Germany) was used to estimate the DNA concentration [19].

\section{2.4. PCR Amplification for DGGE}

121 The fecal bacterial DNA was used for PCR-DGGE. Universal linkage primers table 1was

122 deployed to augment $16 \mathrm{~S}$ rRNA gene focusing V3 region. $50 \mu 1$ PCR reaction mixture was 123 amplified through PCR touchdown programming by using thermocycler (ABI2720 USA):

124 final PCR products were electrophoresed on 1.5\% agarose gel and stained with ethidium 125 bromide for visualization under UV illumination [20]. 


\subsection{Denaturing gradient gel electrophoresis}

127 Denaturing gradient gel electrophoresis was performed by using the DCodeTM Universal

128 Mutation Detection System (Bio-Rad, Hercules, CA, USA). Briefly, amplified PCR

129 product from total bacteria was loaded in $8 \%(\mathrm{w} / \mathrm{v})$ polyacrylamide (acrylamide-bis, 37.5:1)

130 gels in the $1 \times \mathrm{TAE}$ buffer, containing 30 65\% linear denaturant gradient. The gel was

131 allowed to run for 14 hours at $90 \mathrm{~V}$ at a constant temperature of $60^{\circ} \mathrm{C}[21]$

\subsection{Statistical analysis of DGGE band pattern}

133 Bacterial diversity was estimated by the number of bands and band intensity of DGGE 134 profiles by applying Syngene software (Bio-Rad, USA). The diversity of taxa was 135 evaluated by Shannon-Weaver index of diversity $\left(H^{1}\right)[22,23]$. Similarity matrix and cluster 136 analysis of DGGE profiles were computed by using the UPGMA method based on the Dice 137 similarity coefficient (band-based). Microsoft Excel 2010 and GraphPad 7 prism were 138 applied, whereas $(\mathrm{P}<0.05)$ was considered as statistically significant. Similarities among 139 the samples were shown through graphical dendrogram (Fig.1.B and Fig.1.D). Clustering 140 algorithm and (UPGMA) arithmetic averages weredeployed to estimate unweighted pair 141 group dendrograms [24].

142 Shannon Weaver diversity index $\left(H^{1}\right)$ was estimated with the help of the following equation.

$$
\text { Shannon-Weaver index }\left(H^{1}\right)=-\sum_{i=1}^{S}(P i)(\operatorname{In} P i)
$$

\section{Excision of bands and sequencing}

145 Physically a sterilized scalpel was used to excise the dominant band of interest from the 146 gel with care. The polyacrylamidegel piece was placed in a $2 \mathrm{ml}$ tube containing50 $\mu$ lof 147 water and incubated at $37^{\circ} \mathrm{C}$ for $30 \mathrm{~min}$. After centrifugation, $8 \mu \mathrm{l}$ of this was used as a 148 template for PCR re-amplification (targeting V3 region) with the same primers (without 
149 GC-clamps) as previously used for DGGE analysis[25]. ABI 3500xL was used to 150 sequencing the reamplified PCR products. Sequences were studied and analyzed by using

151 BLAST and Seqmatch software for identification of species or genus.

\section{$152 \quad$ Real time PCR execution}

153 Real time PCR was performed in Bio-Rad CFX96 (USA) system. Total $20 \mu$ PCR reaction 154 combination possessed $1 \mu \mathrm{l}$ of two linkage primer $(5 \mathrm{uM}), 10 \mu \mathrm{l} 2 \times \mathrm{SYBR}$ Green 155 (TOYOBO, Japan), $2 \mu$ l sample DNA and $6 \mu$ sterilized $\mathrm{H}_{2} \mathrm{O}$ [26]. Real time PCR Primers 156 are shown in (Table 1). Clostridium leptum(YIT.6169), Bacteroides vulgatus NWS 157 Lactobacillus, (from our lab), (CICC.22938) and Bifidobacteriaum(CICC.6186) were 158 taken as standard strains. Real time PCR was performed in thrice and mean was calculated. 159 The outcome data were considered as the estimate of average logarithms in afecal sample 160 of PCR genomic amplicons, replica counts in $1 \mathrm{~g}$ of fecal mass.

$161 \quad$ 2.7.Pyrosequencing and data analysis

162 Twenty fecal samples were randomly selected for metagenomic pyrosequencing analysis

163 (10 samples from HT and 10 samples from healthy control). According to a previously 164 described method, 16S rRNA gene along with V4 location was augmented with linkage 165 primer: $\quad 515 \mathrm{~F} \quad$ (GTGCCAGCMGCCGCGGTAA) 166 (GGACTACHVGGGTWTCTAAT) primers to develop the amplicon libraries [27]. The 167 sequencing along with paired-end was performed on the platform with an Illumina Miseq 168 based on a standard protocol from the manufacturer. Raw data were screened and 169 assembled by QIIME [28] and FLASH [29] software packages. The UCLUST method 170 [28]was applied in clustering the bacterial sequences in OTUs (Operational Taxonomic 171 Units) at an identity threshold of 97\%. Meanwhile, RDP Classifier [30] was applied to allot 
172 each OTU to a taxonomic level. Diversity analysis, such as Shannon and Simpson diversity

173 index, Chao1, ACE and Good's coverage, was carried out with QIIME. In addition, the

174 OTU table produced by the QIIME pipeline was imported into MEGAN 4 and mapped on

175 the NCBI taxonomy database [31]. The significant statistical differences of gut microbial

176 community texture along with Simpson and Shannon index between DNA sample batches

177 were estimated by computing (unpaired nonparametric $t$ test) Microsoft Excel 2010 and 178 statistic software GraphPad Prism 7.

$179 \quad$ 3. Results

\subsection{Statistical DGGE characterization of bacterial population in HT}

181 The Denaturing Gradient Gel Electrophoresis (DGGE) was deployed with amplified PCR 182 product targeting $16 \mathrm{~S}$ rRNA gene along with specific primers at the site of $\mathrm{V} 3$ region in 183 both HT patients and control. The findings in figure 1 panel A (H1-H17), indicate samples 184 from HT and (C1-C6) healthy control, while figure 1 panel C (H18-H29), with samples 185 from HT and (C7- C12) healthy control. The band's intensity, location, and number were 186 diverse among samples indicating diverse intestinal microbial fingerprints. Syngene 187 software detected the sums of 278 bands in 29 tracks of HT with an average band of (9.2 $188 \pm 3.75)$. A total 96 bands were detected in 12 tracks of the control group with an average 189 of $(8.00 \pm 2.04)$. Stool microbial diversity among patients and normal control group were 190 analyzed by applying nonparametric unpaired $t$ test to evaluate the $\left(H^{1}\right)$ Shannon Weaver 191 diversity index. The $\left(H^{1}\right)$ diversity outcomes depicted $(2.72 \pm 0.621$ vs. $2.64 \pm 0.45)$ 192 insignificant $(\mathrm{P}=0.299)$ gut microbial diversity difference between $\mathrm{HT}$ and healthy control 193 groups. Conversely while comparing the Shannon Weaver index $\left(H^{1}\right)$, it was found to be 194 higher in HT group in contrast to control group that denoted the bacterial overgrowth in 
195 the diseased group. The DGGE profiles similarity levels of all samples were determined 196 by (UPGMA) dendrogram and Dice similarity coefficient (figure 1 panel B, D). The value 197 of band based assessment along with Dice similarity coefficient in HT and healthy control, 198 through mean similarity index, were $(0.300 \pm 0.280)$ and $(0.290 \pm 0.121)$, respectively 199 shown in (table.2). Comparative statistical samples estimated values in HT and control 200 groups were evaluated by using Dice similarity coefficient as well as mean similarity index 201 linking between the two groups. The results indicated the lower level of similarity index in 202 inter-group in contrast to intra-group that demonstrated the gut microbiota of HT patients 203 were differentfrom healthy control group.

204

205

206

207

208

209

210

211

212

213 
A

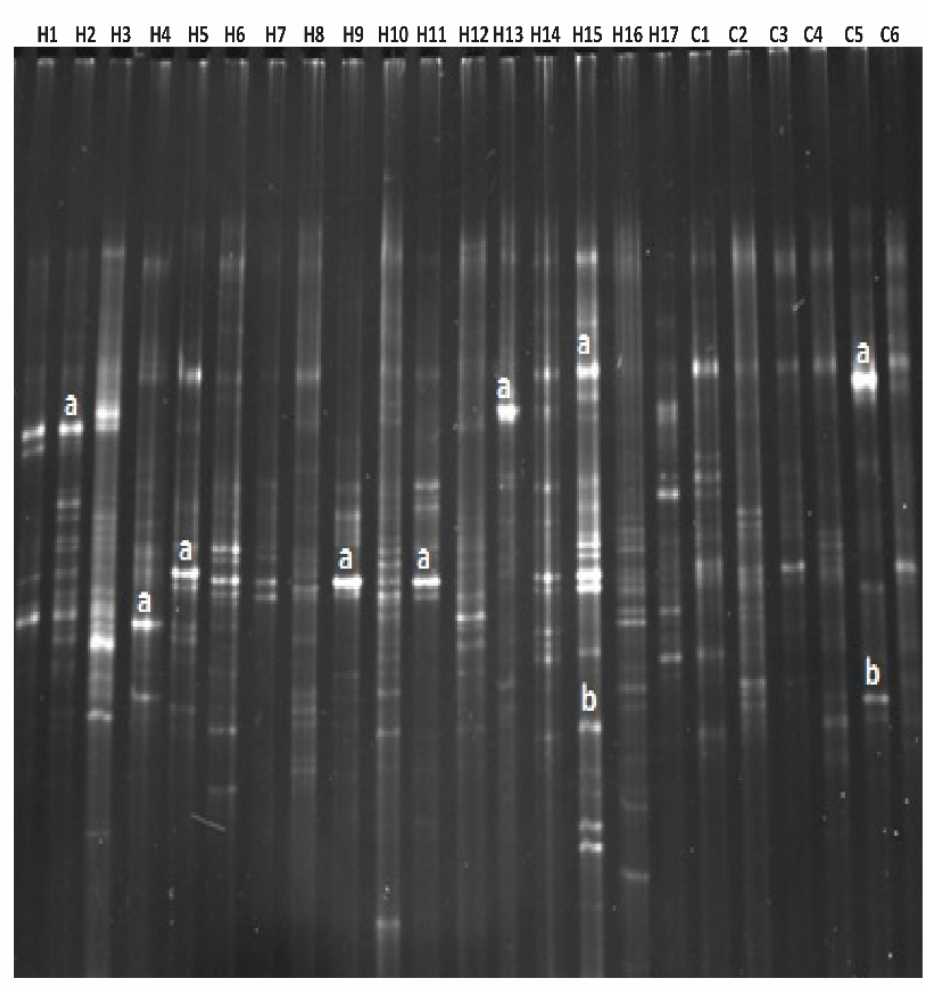

C

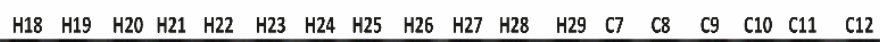

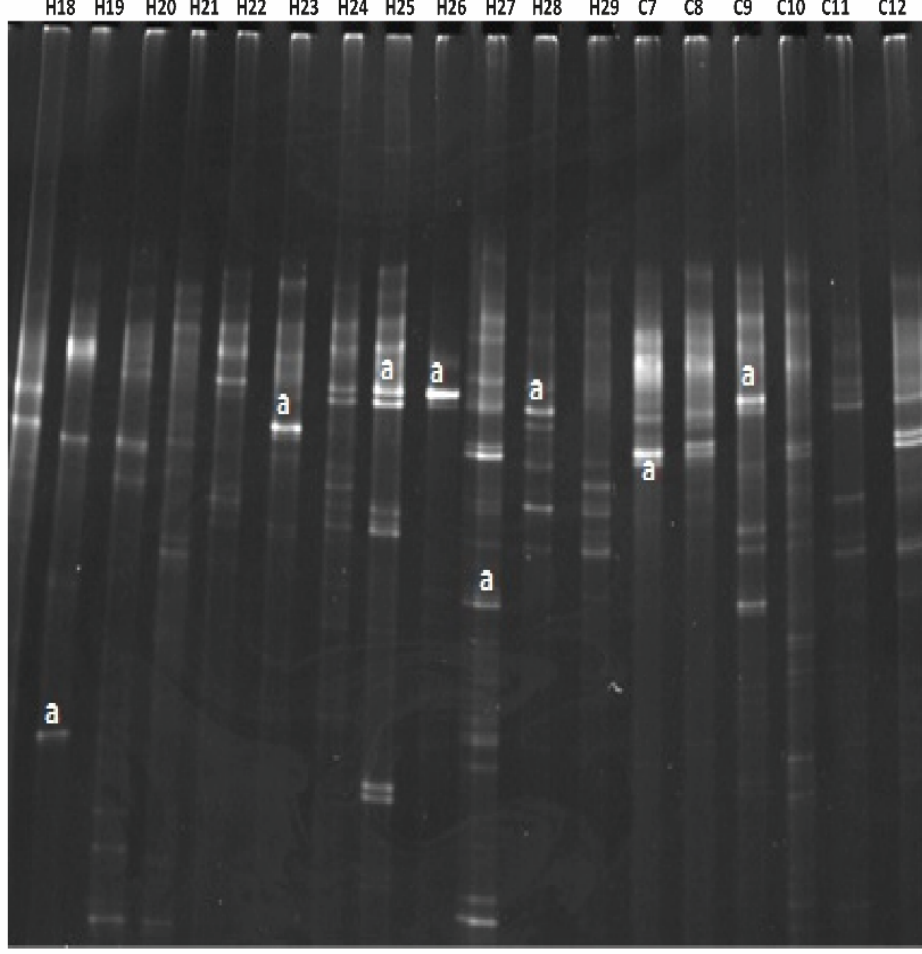

B

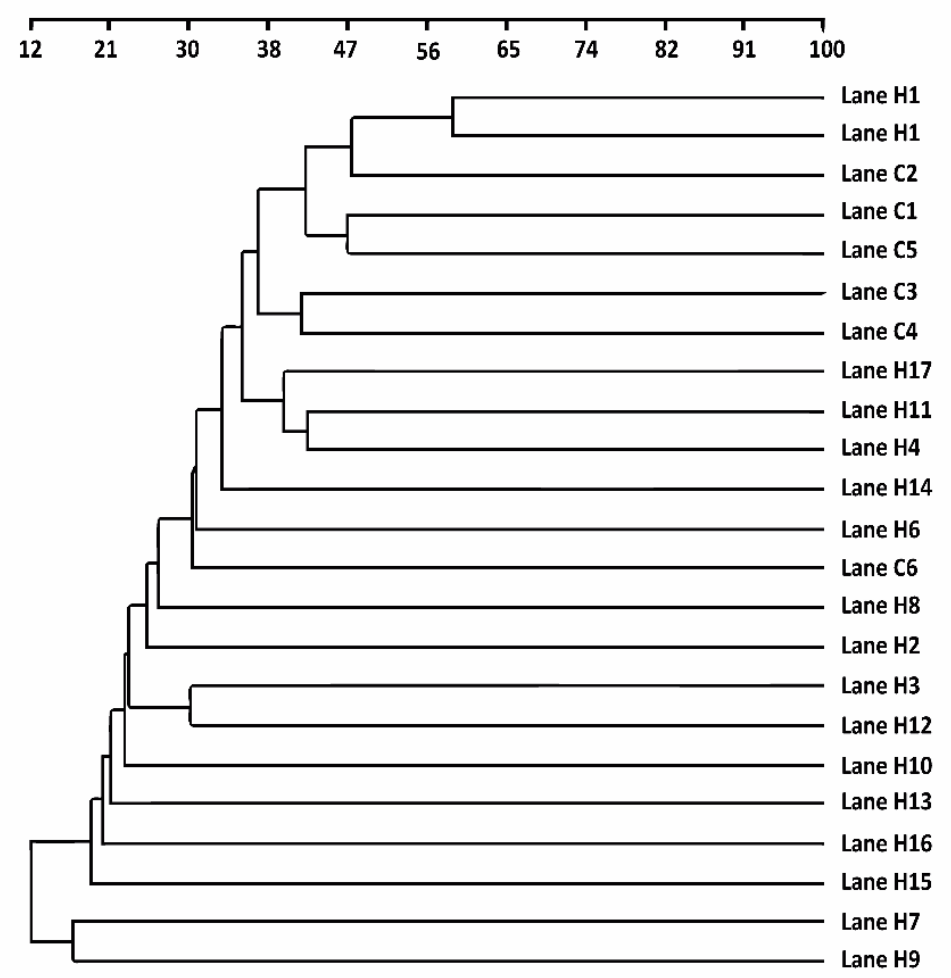

D
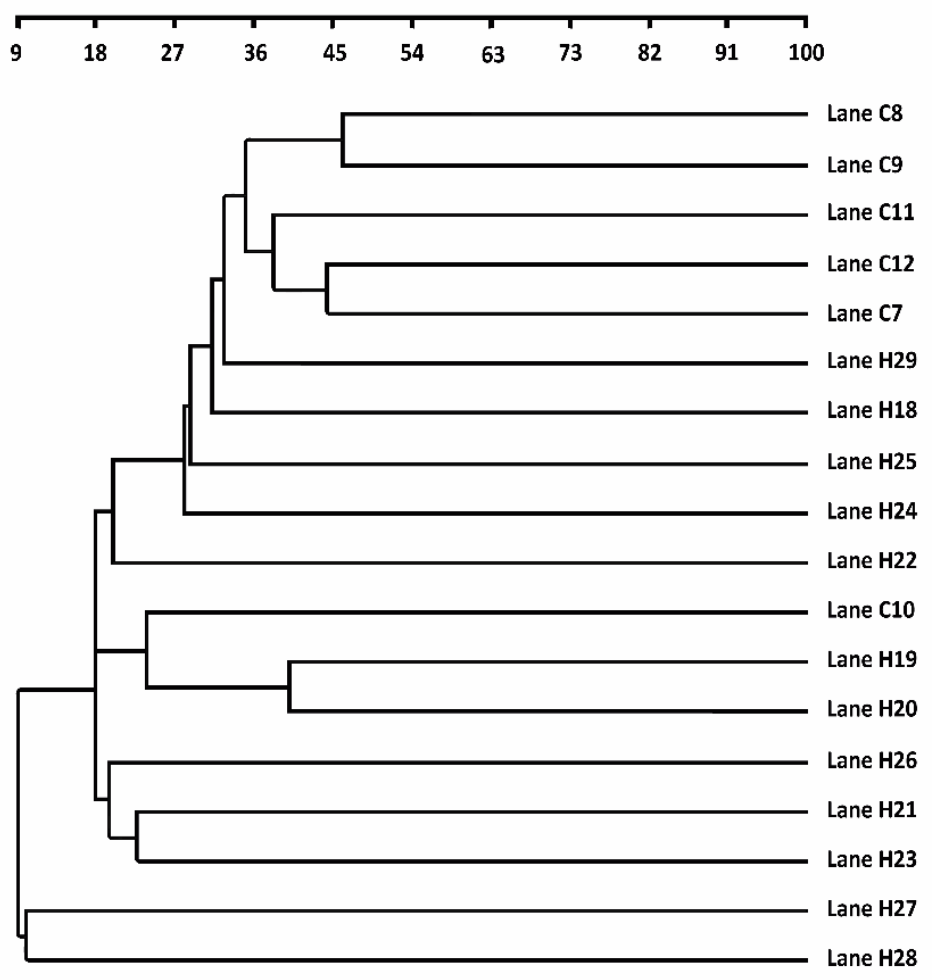
216 Figure 1., (A) DGGE profile constructed betweenHT (H1-H17) and control groups (C1-

217 C6). (B) Cluster analysis between HT(H1-H17) and control (C1-C6) groups by applying 218 UPGMA. (C) DGGE profile erected between HT (H18-H29) and control groups (C7219 C12).(D) Cluster analysis between HT (H18-H29) and control (C7-C12) groups by 220 applying UPGMA. "a" and "b"in figure (A) and (C) related to dominant bands from different 221 patients. $\mathrm{H}$ or $\mathrm{C}$ represent Hashimoto and control group, respectively.

\subsection{Sequence results analysis in DGGE}

223 A total of 18 bands were excised from two DGGE gels. From DGGE gel figure 1panel A, 22410 bands were cut for gut bacterial quantity estimation. To endorse the resolution 225 competence of DGGE genomic bands in the same positions but indifferent lanes (H9a and 226 H11a) were excised and sequenced. Bands H9a and H11a were identified as Shigella 227 dysenteriaewith98\% similarity. Similarly, from (figure 1 panel C), 8 bands were cut. Also 228 to check the resolution ability of DGGE gel, bands H25a, H26a were sequenced and 229 identified as Shigella flexneriwith 99\%similarity. Taxonomic identity of other genomic

230 bands are shown in table.3. The results were demonstrating the phylum Proteobacteria 231 Firmicutes and Bacteroidetes were prevalent in all samples. The findings of two DGGE gel 232 profile were also illustrated, the prevalence of opportunistic gut bacteria (Bacteroides 233 uniformis, Bacteroides pyogenes, Bacteroides vulgates, Shigella dysenteriae, Bacteroides 234 intestinalis, Escherichia coli, Sporomusa ovate, Bacillus sp., Shigella flexneri)in HT 235 patients.

$236 \quad 3.3$. Real time PCR 
237 By applying real time PCR, Bifidobacterium, Clostridium leptum,Bacteroides vulgatus and

238 Lactobacillus were quantified, the resultant copy number of Bifidobacterium and

239 Lactobacillus were significantly $(\mathrm{P}<0.05)$ reduced in the diseased group as compared to

240 healthy subjects. On the other hand, the replica count of Bacteroides vulgatuselevated non-

241 significantly and Clostridium leptumwas lowered non-significantly in the patients while

242 comparing to control group, respectively. All these results were summarized in table.4.

243 3.4. Pyrosequencing analysis of gene sequence

244 The PCR sequence amplicons comparative statistics were estimated with 1,843,183 at 245 theV4 site of $16 \mathrm{~S}$ rRNA gene from $10 \mathrm{HT}$ and 10 from a normal control. Among these sum 246 of pyrosequencing reads 1,541,154 (control 767,213 and disease 773,941, with an average 247 per sample 77,058) were passed for quality control and were processed for further analysis. 248 Taxon tag was (Ave. 72810.35) in all samples of both HT and control group and the total 249 unique tag count detected in diseased and control group was 12241 and10457, respectively 250 (with Ave. 1134.9 in all samples).The total number of OUT were assigned 5509 (control 2512644 and disease 2865, with an average per sample 275.45) in this study. The sum of the 252 unique tag from the two groups was 22698 that exhibited the whole phylotypes in the 253 current study, after deletion of linkage primers; the length of the average sequence was 418 254 bp.

255

\subsection{Gut microbial diversification and conformational analysis}

257 The richness and diversity of bacterial community were calculated at the $97 \%$ similarity 258 level. Alpha diversities, as estimated by nonparametricalgorithm ACE, Chao1 and 259 observed species were significantly elevated in HT $(\mathrm{P}=0.042, \mathrm{P}=0.039)$ andP= 
260 0.045respectively)as comparedto the normal individuals. However, Good's coverage was 261 significantly higher in control group $(\mathrm{P}=0.012)$. Conversely, there were no statistically 262 significant differences in Simpson and Shannon diversity index between the two groups. 263 The degree of diversity estimation in all thesame groups of bacterial community is shown 264 in table 5. Furthermore, the alpha gut bacterial diversity analysis demonstrates the raised 265 level in HT patients as compared to control group. The diversity elevation indicates a clear 266 gut microbial overgrowth in patients group in contrast to healthy control. The bacterial 267 DNA samples within each group or individual samples were divided into two clusters, 268 based on weighted UniFrac metrics depicted in figure 2 corresponding to samples of HT 269 and normal control group analogous to the pattern of PCR-DGGE. 


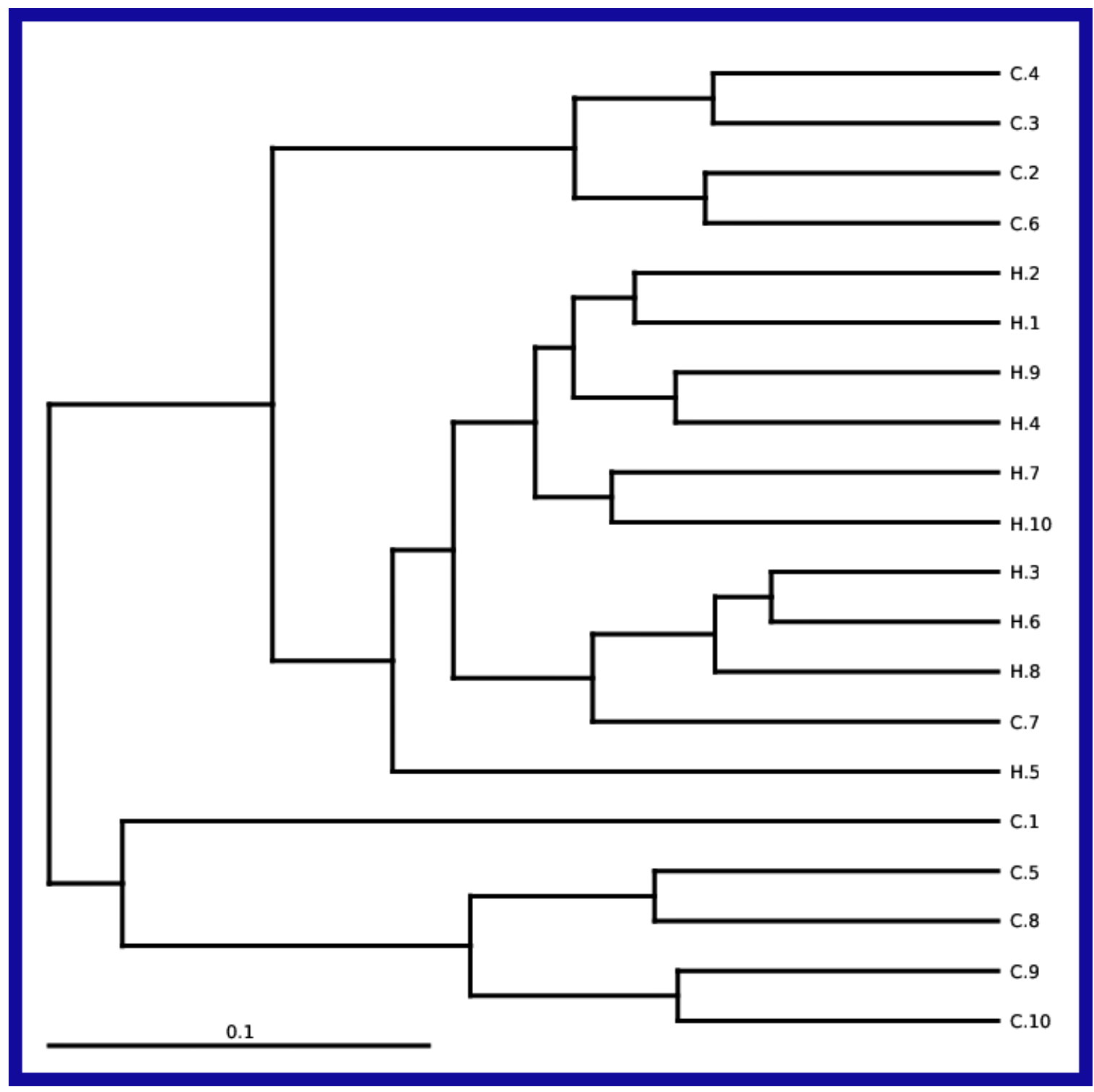

271 Figure 2. Diversity among HT samples of pyrosequencing. UPGMA based on weighted

272 UniFrac distances.H or C represent Hashimoto and control group, respectively.

274 a) Gut microbiota population at phyla level

275 The gut microbial taxa composed of more than (1\%-0.5\%) were focused, and composition

276 was assessed on the taxonomic basis at phylum, family, genus and species level. 
277 At Phylum level, total 13 phyla were sequenced;depicted intable S1, among the 10 top 278 most phyla, the more phyla abundance of Proteobacteria and Cyanobacteria and less 279 Firmicutes and Bacteroidetes in the study group as compared to the normal healthy 280 controls, shown in figure 3. The data statistics in table6 demonstrated that dissimilarity 281 was quantitatively different in top 10 phyla between study and control groups.

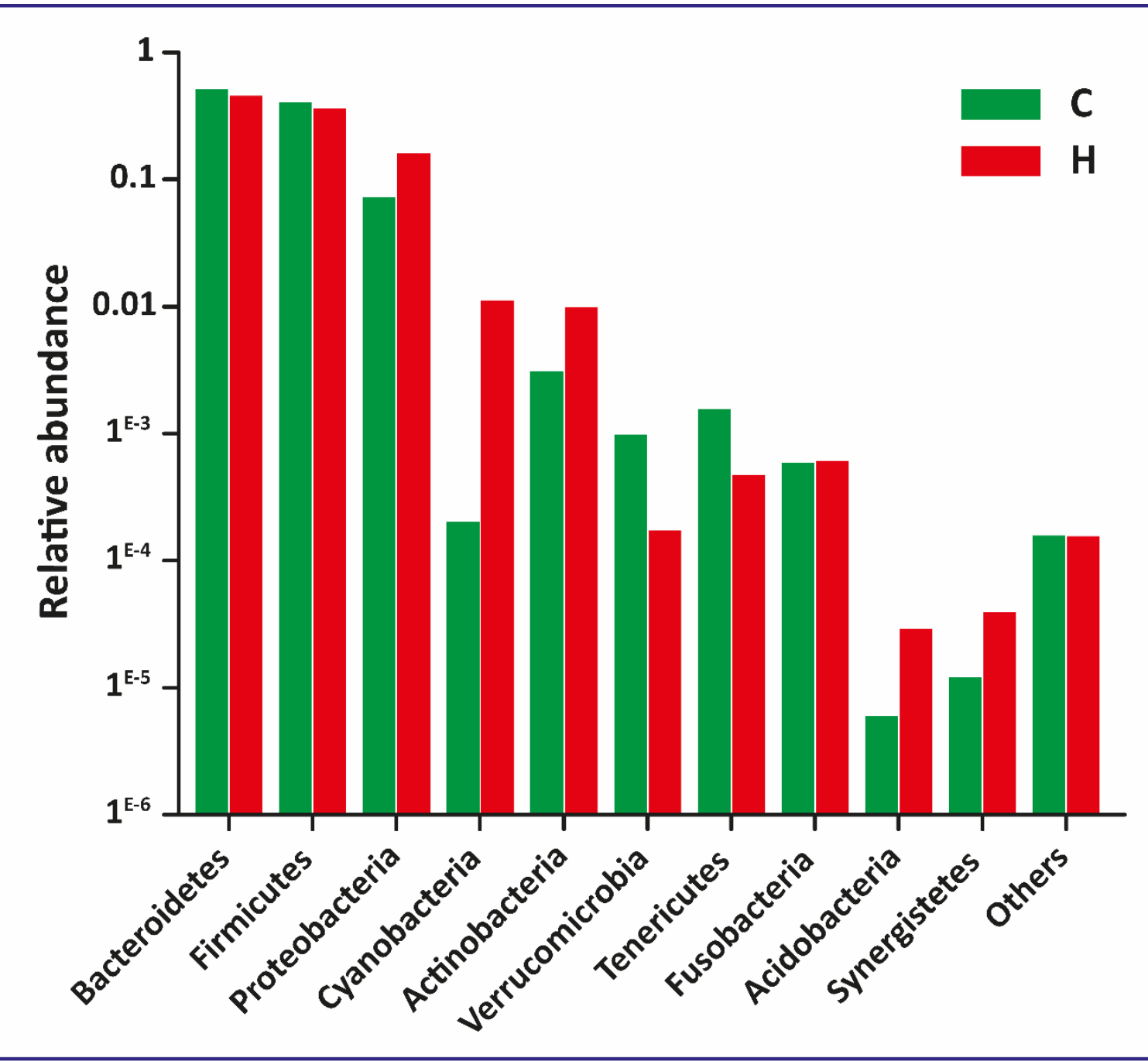

283 Figure 3.Gut microbial composition at phyla levels from Pyrosequencing results. Relative

284 abundance of most prevalent phyla in HT and healthy control.H or C represent Hashimoto 285 and control group, respectively

b) Gut microbial organization at family level 
287 At the family level, 83 different families were sequenced, among 10 top most families, the 288 prevalence of Bacteroidaceae, Enterobacteriaceae and Alcaligenaceae were higher in the 289 diseased group as compared to control, shown in figure4. In these families, the relative 290 abundance of Prevotellacea, Ruminococcaceae, and Veillonellaceae was lowered in 291 patients group in contrast to healthy control. The family level data statistics with the 292 percentage in HT illustrates the quantitative difference displayed in table 6.

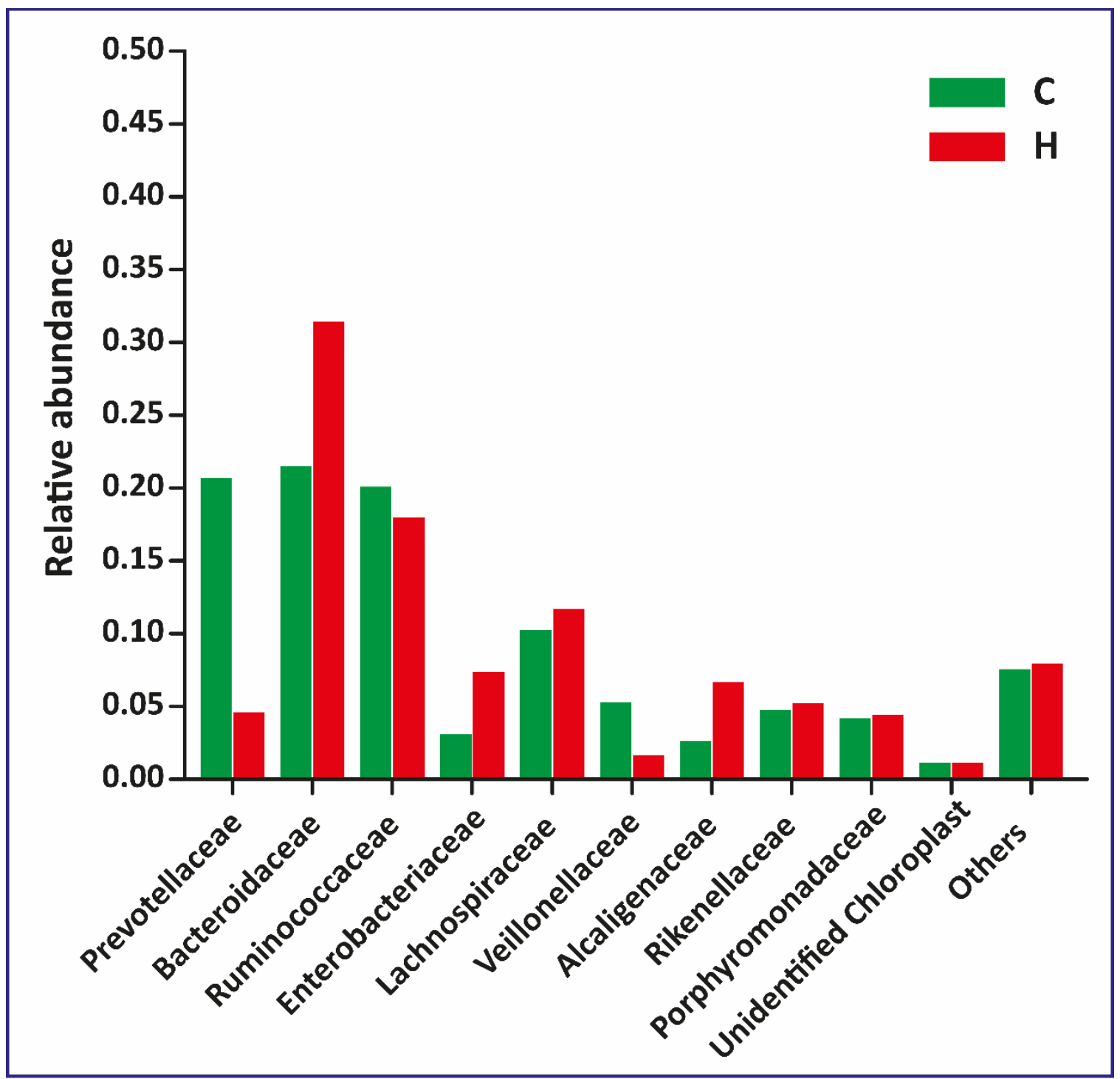


294 Figure 4. Pyrosequencing results of gut microbial compositions at family levels. Relative 295 abundance of most dominant families in HT and healthy controls.H or C represent 296 Hashimoto and control group, respectively

\section{7 c) Gut microbiota distribution at Genus level}

298 The genus level sequence represented with 194 different genera. In 10 top most genera, 299 there was raised anabundance of Bacteroides, Escherichia-Shigella and Parasutterella 300 genera in the diseased group in contrast to control group shown in figure 5. However, 301 decreased genera in disease group were Prevotella_9 and Dialister. The Statistics 302 dissimilarity at genera level in HT group findings were compiled in table 6.

303 HT has an impact on specific groups of gut microbiota, in particular, the Phylum 304 Proteobacteria, family Prevotellaceae, Veillonellaceae, generaPrevotella_9, Dialister, and 305 Escherichia-Shigella and also largely influences the gut flora, which diverges as of the 306 general normal healthy status based on the intestinal microbial composition of the 307 individual. 


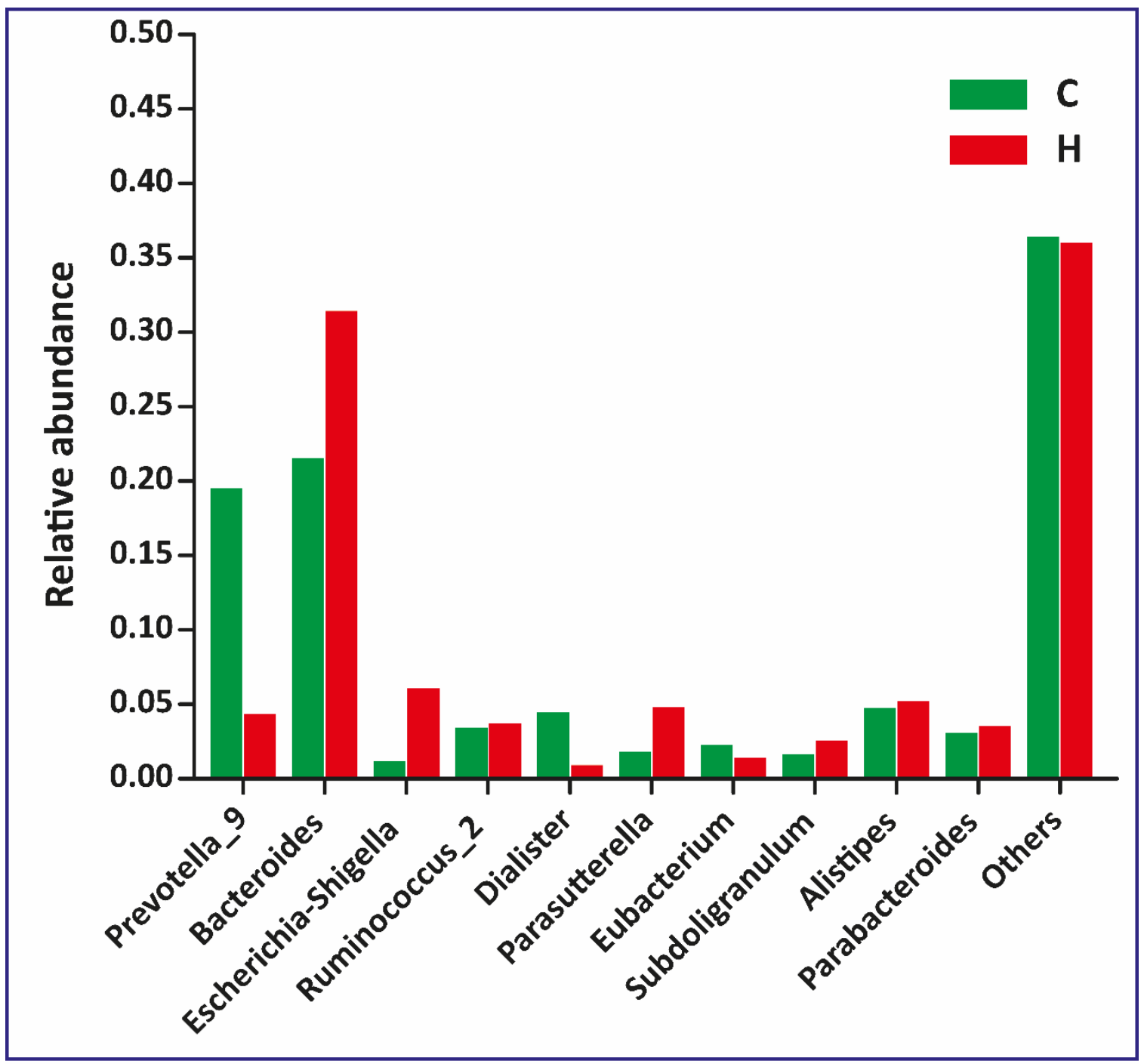

Figure 5. Gut microbial compositions at genera levels from Pyrosequencing results.

Relative abundance of most prevalent genera in HT and healthy control.H or C represent Hashimoto and control group, respectively

313 The patterns of gut microbial community monitored at species level shown in table 7.

314 These findings demonstrate the considerable dissimilarity at species level between HT and 315 control group.However, E.coli has raised the level in HT patients in comparison to their 316 healthy controls. 
317 The concluding data from these findings by applying the metagenomic analysis of DGGE

318 and pyrosequencing validate the same prevalent bacterial taxa. Nevertheless, the much

319 reliable and sensitive pyrosequencing procedure affirm the authenticity of enhanced

320 diversified bacterial population than DGGE. Quantitatively, trend wise metagenomic

321 pyrosequencing and Real time PCR results ratified each other in the whole bacterial

322 community. Therefore, in conclusion, the whole result findings were thus aligning the gut

323 microbiota data produced by the three molecular methods.

\section{4. Discussion}

325 Human gut microbiota plays a critical role inbody protection through metabolic, trophic 326 and protective function [32]. The gut microbial composition can be altered in disease 327 conditions like Crohn's disease, malnutrition, inflammatory bowel disease, colitis, obesity 328 and type II diabetes [16]. The experimental findings have elaborated that there is a distinct 329 difference between the gut microbial composition of HT patients and healthy controls. The 330 results were validated by revealing the dominant bands sequencing of DGGE profile, 331 pyrosequencing analysis as well as real time PCR. The statistical analysis of $\alpha$-diversity, 332 nonparametric Chao1, algorithm ACE, and observed species was significantly elevated in 333 the diseased group as compared to healthy control, while good's coverage was significantly 334 higher in control group. Conversely, no significant distinction was found in diversity index 335 like Shannon and Simpson, which was aligned with recently reported work[33]. Moreover, 336 the bacterial community diversity estimation by DGGE profiles and pyrosequencing 337 analysis was higher in HT patients. This elevation denotes the overgrowth of the gut flora 338 in patients than in healthy control, although there was raised interpersonal difference that 
339 corresponds to previous microbial finding in the vagina, gastrointestinal tract and skin [34, $34035]$.

341 The statistical interpretation of similarity index of gut microbiota in HT patients in DGGE 342 profiles pattern in intra-groups was found to be higher; this rise clearly demarcated the 343 intestinal bacterial overgrowth in patients group. The estimation of similarity index 344 comparison i.e. less in inter- group as compared to intra-group that by previous research 345 findings[36], demonstrating the dissimilarity of gut microbial composition in HT patients 346 as compared to control group. Therefore, all these diversity above outcomes illustrate that 347 there is a significant disparity of gut microbial texture between diseased and control group. 348 The statistical data represent the important quantitative difference between diseased and 349 control groups. At the phylum level, Actinobacteria showeda raised level in the diseased 350 group as compared to control which is consistent with previous work of physiological stress 351 and gut microbiota [37]. The relative abundance of families Prevotellaceae and 352 Veillonellaceae was lowered in patients group in contrast to healthy control which is 353 aligned with previous work $[33,38]$.Veillonellaceae has beneficial commensal role i.e.very 354 closely interrelated toClostridium and isinvolved inthe induction of immune T regulatory 355 cells [39]. While Enterobacteriaceae and Alcaligenaceae were higher in the diseased group 356 in comparison to healthy control.A Higher level of Enterobacteriaceae is aligned with 357 previous work of type 2 diabetic patients and gut flora [40].However, decreased genera in 358 the diseased group were Prevotella_9 and Dialister. The diminished Prevotella presence 359 has been shown in a disease like autism and types1 diabetes with intestinal microbiota 360 [41, 42], while augmented genera in the diseased group were Escherichia-Shigella and 361 Parasutterella. The raised level of Escherichia-Shigellais reported in preceding work of 
362 autism spectrum disorders related to intestinal flora [43], while a higher level of 363 Parasutterella aligned with preceding findings[44].

364 The current meta-analysis on intestinal microbiota linked with obesity and IBD revealed 365 that percentage between Bacteroidetes to Firmicutes: may not be a steady characteristic 366 that is distinctive between obese to lean gut flora[45]. Also, the current study findings 367 demonstrate the raised level of Actinobacteria. It has been documented that Actinobacteria 368 has the capability to settle in gingival crevices and is responsible for dental plaque 369 accumulation, also produces the acid that results in infections or cavities [46].Our findings 370 denoted the decreased values of genus Prevotella;nevertheless, the literature evidence 371 describes the dominance of Prevotella in agut microbial composition exhibiting the 372 positive impact on host metabolism[47]. Prevotella prevalence is considered as abeneficial 373 bacteria in connection with plant based diet, and thus its intestinal flora has linked with 374 many diseases and inflammatory conditions $[48,49]$. The results showed a raised level of 375 Escherichia-Shigella in HT patients as compared to healthy subjects. It has been 376 documented that Escherichia-Shigella is Shiga-toxin producing bacteria that can cause 377 septicemia, hemorrhagic colitis, thrombocytopenia, severe gastrointestinal tract 378 inflammations in particular ileo-colonic area, spiteful problems of urinary duct channels 379 and (HUS) hemolytic uremic syndrome.[50]. The current experimental study depicted a 380 raised level of genus Escherichia-Shigella, in particular, species (E.coli) that might be the 381 causative gut microbe in HT. Furthermore, ubiquitous Escherichia coli are responsible for 382 causing the prevalent infections like (UTIs) urinary tract infections and food borne illnesses $383[51]$ 
384 The evidence of current work elaborated the relative predominance of phylum, family,

385 genus as well as species level taxa in stool samples, which also illustrated a clear disparity

386 between HT patients and normal healthy subjects. Moreover, species level phylotypes with

387 community comparison also divulged a clear demarcation of intestinal microbial texture

388 between diseased and control groups [52]. These investigations further unravel the HT role

389 in physiological intestinal changes that in turn contributes in alteration of the gut microbial

390 composition. Moreover, these fluctuations in gut microbial configuration may lead to the

391 complication of diseases[53].

392 The clinical signs of autoimmune HT manifested with hypothyroidism, goiter along with

393 circulating antibodies to thyroid antigens. The results of the serum thyroid hormones level

394 and circulating antibodies, anti-thyroglobulin anti-thyroid peroxidase in both HT and

395 control, shown in table S3 and table S2 respectively. The findings of HT patientsin table

396 S3 demonstrate the extremely raised level of aforesaid antibodies in HT as compared to

397 healthy subjects. The increased level of antibodies in HT patients might change the gut

398 microbial composition, in particular, the PhylumProteobacteria, family Prevotellaceae

399 Veillonellaceae, generaPrevotella_9, Dialister,Escherichia-Shigella and E.coli species,

400 and also broadly affect the gut flora. The current work on gut microbial differences between

401 HT patients and healthy subjects was found pretty motivating as there was no straight and

402 direct established relationship between HT and intestinal flora. Therefore, current findings

403 further elaborate the diversification of gut microbial compositions between HT diseased

404 and healthy groups. These fluctuations may change the health status of the host even though

405 disease development is not associated with the intestinal tract [54]. 
406 The Real time PCR was performed to observe the gut microbial quantitative alterations

407 [55], the data represent a significant decrease in Bifidobacterium and Lactobacillus in the

408 diseased group thus aligning with preceding work [56]. The probiotics used in food most

409 recurrently, generally belong to Bifidobacterium and Lactobacillus genera and encompass

410 physiological benefits in the body[57]. Bifidobacterium and Lactobacillusare constantly

411 reduced in colorectal cancer [58]. In addition to that, they also showed anti-obesity, anti-

412 inflammatory and anti-atherogenic influential effects in many studies [59]. Various

413 lactobacillus strains possess fair antimicrobial behavior to shield the body in opposition to

414 uropathogens[60].

415 The current study findings generated from DGGE and pyrosequencing are reliable to 416 analyze the gut microbiota. However, an experimental technique like PCR-DGGE is a 417 semi-quantitative procedure; outcomes of bands density evaluation might not narrate the 418 target abundance of gut flora correctly. [23]. However, pyrosequencing is a more advanced, 419 sensitive and more reliable method to investigate and analyze the gut microbial ecology 420 [54]. On the other hand, thePCR-DGGE technique could be used as a basic test to examine 421 the notable shift of gut microbial community due to inexpensive and less time422 consumingprocedure.

\section{5. Conclusion}

424 The current study demonstrates that gut microbial composition is different between HT 425 patients and the normal control groups. More precisely, there is an important dissimilarity 426 of gut microbial taxa richness as compared to control group. Furthermore, thelevel of 427 certain intestinal microbes was either lowered or elevated profusion in HT patients in 428 comparison to their healthy counterparts. The diversity of bacterial community estimation 
analysis demonstrates an elevated level of gut flora in HT patients as compared with

430 controls, which is an indication of bacterial overgrowth in HT patients. Therefore, the

431 further multicenter approach is obligatory to comprehend the underlying mechanism and

432 process of intestinal bacterial dysbiosis in Hashimoto's thyroiditis.

\section{Disclosure}

434 All authors disclose that they do not have any conflict of interest.

435 Acknowledgement

436 The author would like to thank Dr. Hui Guo (Department of Endocrinology 1st affiliated

437 Hospital Xi'an Jiotong University, China) for providing support in sample collection for

438 this study.

439 Literature Cited:

440 [1] S.E. Power, P.W. O'Toole, C. Stanton, R.P. Ross, G.F. Fitzgerald, Intestinal microbiota, diet and 441 health, British Journal of Nutrition (2013) 1-16.

442 [2] C.J. Walsh, C.M. Guinane, P.W. O'Toole, P.D. Cotter, Beneficial modulation of the gut

443 microbiota, FEBS Letters (2014).

444 [3] N. Kamada, G.Y. Chen, N. Inohara, G. Núñez, Control of pathogens and pathobionts by the 445 gut microbiota, Nature immunology 14(7) (2013) 685-690.

446 [4] Y. Hiromatsu, H. Satoh, N. Amino, Hashimoto's thyroiditis: history and future outlook, 447 Hormones 12(1) (2013) 12-8.

448 [5] D.S.A. McLeod, D.S. Cooper, The incidence and prevalence of thyroid autoimmunity, 449 Endocrine 42(2) (2012) 252-265.

450 [6] S.H. Golden, K.A. Robinson, I. Saldanha, B. Anton, P.W. Ladenson, Prevalence and Incidence 451 of Endocrine and Metabolic Disorders in the United States: A Comprehensive Review, Journal of 452 Clinical Endocrinology \& Metabolism 94(6) (2009) 1853.

453 [7] B. Delemer, J.P. Aubert, P. Nys, F. Landron, S. Bouée, An observational study of the initial 454 management of hypothyroidism in France: the ORCHIDÉE study, European Journal of 455 Endocrinology 167(6) (2012) 817-23.

456 [8] P. Caturegli, A.D. Remigis, N.R. Rose, Hashimoto thyroiditis: Clinical and diagnostic criteria, 457 Autoimmunity reviews 13(4-5) (2014) 391-397.

458 [9] W.M. Wiersinga, Smoking and thyroid, Clinical Endocrinology 79(2) (2013) 145-151.

459 [10] J. Cárdenasroldán, A. Rojasvillarraga, J.M. Anaya, How do autoimmune diseases cluster in 460 families? A systematic review and meta-analysis, Bmc Medicine 11(1) (2013) 73.

461 [11] W. Teng, Z. Shan, X. Teng, H. Guan, Y. Li, D. Teng, Y. Jin, X. Yu, C. Fan, W. Chong, Effect of 462 iodine intake on thyroid diseases in China, The New England journal of medicine 354(26) (2006) $463 \quad 2783-93$. 
[12] X. Teng, Z. Shan, Y. Chen, Y. Lai, J. Yu, L. Shan, X. Bai, Y. Li, N. Li, Z. Li, More than adequate iodine intake may increase subclinical hypothyroidism and autoimmune thyroiditis: a crosssectional study based on two Chinese communities with different iodine intake levels, European Journal of Endocrinology 164(6) (2011) 943.

[13] U. Vyas, N. Ranganathan, Probiotics, prebiotics, and synbiotics: gut and beyond, Gastroenterology Research \& Practice 2012(11) (2012) 872716.

[14] L. Xiao, F.S. Muhali, T.T. Cai, R.H. Song, R. Hu, X.H. Shi, W.J. Jiang, D.F. Li, S.T. He, J. Xu, Association of single-nucleotide polymorphisms in the STAT3 gene with autoimmune thyroid disease in Chinese individuals, Functional \& Integrative Genomics 13(4) (2013) 455-461.

[15] F. Guarner, J.R. Malagelada, Gut flora in health and disease, Lancet 361(9356) (2003) 512519.

[16] A. Khoruts, J. Dicksved, J.K. Jansson, M.J. Sadowsky, Changes in the composition of the human fecal microbiome after bacteriotherapy for recurrent Clostridium difficile-associated diarrhea, Journal of clinical gastroenterology 44(5) (2010) 354-360.

[17] K. Mori, Y. Nakagawa, H. Ozaki, Does the gut microbiota trigger Hashimoto's thyroiditis?, Discov Med 14(78) (2012) 321-6.

[18] A.J. Giannini, The Biological foundations of clinical psychiatry, Medical Examination Pub. Co.1986.

[19] P.D. Scanlan, F. Shanahan, C. O'Mahony, J.R. Marchesi, Culture-independent analyses of temporal variation of the dominant fecal microbiota and targeted bacterial subgroups in Crohn's disease, Journal of clinical microbiology 44(11) (2006) 3980-8.

[20] G. Muyzer, E.C. De Waal, A.G. Uitterlinden, Profiling of complex microbial populations by denaturing gradient gel electrophoresis analysis of polymerase chain reaction-amplified genes coding for 16S rRNA, Applied and environmental microbiology 59(3) (1993) 695-700.

[21] Z. Ling, J. Kong, F. Liu, H. Zhu, X. Chen, Y. Wang, L. Li, K.E. Nelson, Y. Xia, C. Xiang, Molecular analysis of the diversity of vaginal microbiota associated with bacterial vaginosis, BMC genomics 11(1) (2010) 488.

[22] G.P. Gafan, V.S. Lucas, G.J. Roberts, A. Petrie, M. Wilson, D.A. Spratt, Statistical analyses of complex denaturing gradient gel electrophoresis profiles, Journal of clinical microbiology 43(8) (2005) 3971-3978.

[23] R.G. Ledder, P. Gilbert, S.A. Huws, L. Aarons, M.P. Ashley, P.S. Hull, A.J. McBain, Molecular analysis of the subgingival microbiota in health and disease, Applied and Environmental Microbiology 73(2) (2007) 516-523.

[24] N. Fromin, J. Hamelin, S. Tarnawski, D. Roesti, K. Jourdain - Miserez, N. Forestier, S. Teyssier - Cuvelle, F. Gillet, M. Aragno, P. Rossi, Statistical analysis of denaturing gel electrophoresis (DGE) fingerprinting patterns, Environmental Microbiology 4(11) (2002) 634643.

[25] S.J. Green, M.B. Leigh, J.D. Neufeld, Denaturing gradient gel electrophoresis (DGGE) for microbial community analysis, Handbook of hydrocarbon and lipid microbiology, Springer2010, pp. 4137-4158.

[26] T. Matsuki, K. Watanabe, J. Fujimoto, T. Takada, R. Tanaka, Use of 16S rRNA gene-targeted group-specific primers for real-time PCR analysis of predominant bacteria in human feces, Applied and Environmental Microbiology 70(12) (2004) 7220-7228.

[27] J.A. Peiffer, A. Spor, O. Koren, Z. Jin, S.G. Tringe, J.L. Dangl, E.S. Buckler, R.E. Ley, Diversity and heritability of the maize rhizosphere microbiome under field conditions, Proceedings of the National Academy of Sciences of the United States of America 110(16) (2013) 6548-53.

[28] J.G. Caporaso, J. Kuczynski, J. Stombaugh, K. Bittinger, F.D. Bushman, E.K. Costello, N. Fierer, A.G. Pena, J.K. Goodrich, J.I. Gordon, G.A. Huttley, S.T. Kelley, D. Knights, J.E. Koenig, R.E. Ley, 
C.A. Lozupone, D. McDonald, B.D. Muegge, M. Pirrung, J. Reeder, J.R. Sevinsky, P.J. Turnbaugh, W.A. Walters, J. Widmann, T. Yatsunenko, J. Zaneveld, R. Knight, QIIME allows analysis of highthroughput community sequencing data, Nat Methods 7(5) (2010) 335-6.

[29] T. Magoč, S.L. Salzberg, FLASH: fast length adjustment of short reads to improve genome assemblies, Bioinformatics 27(21) (2011) 2957-63.

[30] Q. Wang, G.M. Garrity, J.M. Tiedje, J.R. Cole, Naïve Bayesian Classifier for Rapid Assignment of rRNA Sequences into the New Bacterial Taxonomy, Applied and Environmental Microbiology 73(16) (2007) 5261-5267.

[31] D.H. Huson, S. Mitra, H.J. Ruscheweyh, N. Weber, S.C. Schuster, Integrative analysis of environmental sequences using MEGAN4, Genome Research 21(9) (2011) 1552.

[32] F. Guarner, J.-R. Malagelada, Gut flora in health and disease, The Lancet 361(9356) (2003) 512-519.

[33] J. Chen, N. Chia, K.R. Kalari, J.Z. Yao, M. Novotna, M.M.P. Soldan, D.H. Luckey, E.V. Marietta, P.R. Jeraldo, X. Chen, Multiple sclerosis patients have a distinct gut microbiota compared to healthy controls, Scientific Reports 6 (2016) 28484.

[34] R. Hummelen, A.D. Fernandes, J.M. Macklaim, R.J. Dickson, J. Changalucha, G.B. Gloor, G. Reid, Deep Sequencing of the Vaginal Microbiota of Women with HIV, PloS one 5(8) (2010) e12078.

[35] Z. Ling, J. Kong, P. Jia, C. Wei, Y. Wang, Z. Pan, W. Huang, L. Li, H. Chen, C. Xiang, Analysis of oral microbiota in children with dental caries by PCR-DGGE and barcoded pyrosequencing, Microbial Ecology 60(3) (2010) 677-690.

[36] X. Wu, C. Ma, L. Han, M. Nawaz, F. Gao, X. Zhang, P. Yu, C.a. Zhao, L. Li, A. Zhou, Molecular characterisation of the faecal microbiota in patients with type II diabetes, Current microbiology 61(1) (2010) 69-78.

[37] J.P. Karl, L.M. Margolis, E.H. Madslien, N.E. Murphy, J.W. Castellani, Y. Gundersen, A.V. Hoke, M.W. Levangie, R. Kumar, N. Chakraborty, A. Gautam, R. Hammamieh, S. Martini, S.J. Montain, S.M. Pasiakos, Changes in intestinal microbiota composition and metabolism coincide with increased intestinal permeability in young adults under prolonged physiologic stress, American journal of physiology. Gastrointestinal and liver physiology (2017) ajpgi 000662017. [38] M. Zhang, M. Zheng, Z. Wu, M. Guan, S. Liu, W. Zhao, J. Cheng, Alteration of gut microbial community after N,N-Dimethylformamide exposure, The Journal of toxicological sciences $42(2)$ (2017) 241-250.

[39] K. Atarashi, T. Tanoue, T. Shima, A. Imaoka, T. Kuwahara, Y. Momose, G. Cheng, S. Yamasaki, T. Saito, Y. Ohba, Induction of Colonic Regulatory T Cells by Indigenous Clostridium Species, Science 331(6015) (2011) 337.

[40] Y. Wang, X. Luo, X. Mao, Y. Tao, X. Ran, H. Zhao, J. Xiong, L. Li, Gut microbiome analysis of type 2 diabetic patients from the Chinese minority ethnic groups the Uygurs and Kazaks, PloS one 12(3) (2017) e0172774.

[41] D.W. Kang, G.P. Jin, Z.E. Ilhan, G. Wallstrom, J. Labaer, J.B. Adams, R. Krajmalnikbrown, Reduced Incidence of Prevotella and Other Fermenters in Intestinal Microflora of Autistic Children, PloS one 8(7) (2013) e68322.

[42] M. Murri, I. Leiva, J.M. Gomez-Zumaquero, F.J. Tinahones, F. Cardona, F. Soriguer, M.I. Queipo-Ortuño, Gut microbiota in children with type 1 diabetes differs from that in healthy children: a case-control study, BMC Medicine 11(1) (2013) 46.

[43] F. Strati, D. Cavalieri, D. Albanese, C. De Felice, C. Donati, J. Hayek, O. Jousson, S. Leoncini, D. Renzi, A. Calabro, C. De Filippo, New evidences on the altered gut microbiota in autism spectrum disorders, Microbiome 5(1) (2017) 24. 
[44] E.E. Noble, T.M. Hsu, R.B. Jones, A.A. Fodor, M.I. Goran, S.E. Kanoski, Early-Life Sugar

560 Consumption Affects the Rat Microbiome Independently of Obesity, The Journal of nutrition

561 147(1) (2017) 20-28.

562 [45] W.A. Walters, Z. Xu, R. Knight, Meta-analyses of human gut microbes associated with

obesity and IBD, FEBS letters 588(22) (2014) 4223.

[46] G. Reynolds-Campbell, A. Nicholson, C.-A. Thoms-Rodriguez, Oral Bacterial Infections : Diagnosis and Management, Dental Clinics of North America 61 (2017) 305-318.

[47] P. Kovatchevadatchary, A. Nilsson, R. Akrami, Y.S. Lee, V.F. De, T. Arora, A. Hallen, E. Martens, I. Björck, F. Bäckhed, Dietary Fiber-Induced Improvement in Glucose Metabolism Is Associated with Increased Abundance of Prevotella, Cell Metabolism 22(6) (2015) 971-982. [48] J. Scher, [Original String]: Scher, J.U. et al. Expansion of intestinal Prevotella copri correlates with enhanced susceptibility to arthritis. eLife 2, e01202 (2013), Elife Sciences 2(1629) (2010) e01202.

[49] S.M. Dillon, E.J. Lee, C.V. Kotter, G.L. Austin, S. Gianella, B. Siewe, D.M. Smith, A.L. Landay, M.C. Mcmanus, C.E. Robertson, Gut Dendritic Cell Activation Links an Altered Colonic Microbiome to Mucosal and Systemic T Cell Activation in Untreated HIV-1 infection, Mucosal Immunology 9(1) (2015) 24-37.

[50] J. Amani, A. Ahmadpour, A.A. Imani Fooladi, S. Nazarian, Detection of E. coli O157:H7 and Shigella dysenteriae toxins in clinical samples by PCR-ELISA, The Brazilian journal of infectious diseases : an official publication of the Brazilian Society of Infectious Diseases 19(3) (2015) 27884.

[51] M.A. Mulvey, J.D. Schilling, J.J. Martinez, S.J. Hultgren, Bad bugs and beleaguered bladders: Interplay between uropathogenic Escherichia coli and innate host defenses, Proceedings of the National Academy of Sciences of the United States of America 97(16) (2000) 8829-8835.

[52] C. Manichanh, E. Varela, C. Martinez, M. Antolin, M. Llopis, J. Doré, J. Giralt, F. Guarner, J.R. Malagelada, The gut microbiota predispose to the pathophysiology of acute postradiotherapy diarrhea, American Journal of Gastroenterology 103(7) (2008) 1754-1761.

[53] J. Qin, R. Li, J. Raes, M. Arumugam, K.S. Burgdorf, C. Manichanh, T. Nielsen, N. Pons, F. Levenez, T. Yamada, A human gut microbial gene catalogue established by metagenomic sequencing, Nature 464(7285) (2010) 59-65.

[54] Y.D. Nam, H.J. Kim, J.G. Seo, S.W. Kang, J.W. Bae, Impact of Pelvic Radiotherapy on Gut Microbiota of Gynecological Cancer Patients Revealed by Massive Pyrosequencing, 8(12) (2013) e82659.

[55] S.R. Lyons, A.L. Griffen, E.J. Leys, Quantitative real-time PCR forPorphyromonas gingivalis and total bacteria, Journal of clinical microbiology 38(6) (2000) 2362-2365.

[56] L. Zhou, X. Li, A. Ahmed, D. Wu, L. Liu, J. Qiu, Y. Yan, M. Jin, Y. Xin, Gut microbe analysis between hyperthyroid and healthy individuals, Current microbiology 69(5) (2014) 675-680.

[57] M.J. Butel, Probiotics, gut microbiota and health, Medecine et maladies infectieuses 44(1) (2014) 1-8.

[58] M. Borges-Canha, J.P. Portela-Cidade, M. Dinis-Ribeiro, A.F. Leite-Moreira, P. PimentelNunes, Role of colonic microbiota in colorectal carcinogenesis: a systematic review, Revista Española de Enfermedades Digestivas 107(11) (2015) 659-671.

[59] E. Nova, F. Perez de Heredia, S. Gomez-Martinez, A. Marcos, The Role of Probiotics on the Microbiota: Effect on Obesity, Nutrition in clinical practice : official publication of the American Society for Parenteral and Enteral Nutrition (2016).

[60] Y.H. Shim, S.J. Lee, J.W. Lee, Antimicrobial activities of Lactobacillus Strains against Uropathogens, Pediatrics international : official journal of the Japan Pediatric Society (2016). 
606 